\title{
1 Erkrankungen der Verdauungsorgane in Deutschland
}

\author{
Petra Lynen Jansen, Frank Lammert
}

Die Erkrankungen der Verdauungsorgane umfassen Erkrankungen des Magen-DarmTraktes sowie der Leber, der Gallenblase und der Bauchspeicheldrüse. $\mathrm{Zu}$ den häufigsten gastroenterologischen Erkrankungen zählen die Ulkuserkrankungen, das Kolonkarzinom, die Pankreatitis, Lebererkrankungen (Leberzirrhose, Virushepatitis, Fettlebererkrankung) und Darmerkrankungen wie die Zöliakie oder die chronisch entzündlichen Darmerkrankungen (Morbus Crohn, Colitis ulcerosa). Darüber hinaus sind die Funktionsstörungen (funktionelle Dyspepsie, Reizdarmsyndrom) wegen ihrer Häufigkeit von hoher medizinischer und gesundheitsökonomischer Relevanz. Eine besondere Rolle in der Therapie nimmt die Lebertransplantation ein. Nach der Niere ist die Leber das zweithäufigste Organ, das in Deutschland transplantiert wird.

Die Komplexität des Fachgebiets, das sich anders als z. B. die Pulmologie oder die Kardiologie nicht nur mit einem Organ, sondern mit einem (Verdauungs-)Organsystem befasst, macht seine besondere Attraktivität aus. Sie bedingt aber auch, dass die Häufigkeit gastroenterologischer Erkrankungen in der Öffentlichkeit deutlich unterschätzt wird. Dabei ist das pathologische Prinzip meist ähnlich, beruhend auf einer Kaskade aus Entzündung, Fibrose und Organversagen. Dieses Weißbuch hat das Ziel, die Bedeutung der gastroenterologischen Erkrankungen für das deutsche Gesundheitssystem und für die Volkswirtschaft unseres Landes darzustellen, aber auch das hohe Präventionspotenzial gerade dieses Krankheitsgebiets auszuleuchten.

Allein die nicht-malignen Erkrankungen der Verdauungsorgane (ICD K00-K93) gehören mit zwei Millionen stationären Fällen pro Jahr nach den Herz-Kreislauf-Störungen zu den zweithäufigsten Erkrankungen der Deutschen und liegen damit vor den bösartigen Neubildungen, psychischen Störungen, Atemwegserkrankungen oder Infektionskrankheiten (Abb. 1.1) [1].

Mit mehr als 36.000 Sterbefällen in deutschen Krankenhäusern stellen die Krankheiten des Verdauungssystems (ICD K00-K93) hinter den Krankheiten des Kreislaufsystems, den Neubildungen und den Krankheiten des Atmungssystems die vierthäufigste Todesursache dar (Abb. 1.2) [2].

Die Tatsache, dass die Zahl der Patienten, die mit Erkrankungen der Verdauungsorgane im Krankenhaus behandelt werden, im Zeitraum von 2012 bis 2017 allein im stationären Bereich um 6\% zugenommen hat, untermauert deren gesundheitsökonomische Bedeutung [1]. Die vom AOK Bundesverband veröffentlichte Übersicht über die Arbeitsunfähigkeit der erwerbstätigen Pflichtmitglieder zeigt, dass die Erkrankungen der Verdauungsorgane auf Platz 3 und damit noch vor den Erkrankungen der Psyche und des Herz-Kreislauf-Systems liegen (Abb. 1.3) [3].

2015 wurden die direkten Kosten für Behandlungen gastroenterologischer Krankheiten auf 41,62 Milliarden Euro geschätzt und die Entwicklung der Fallzahlen legt 
Q00-Q99 Angeb. Fehlbild., Deformitäten, Chromosomenanomalien D50-D90 Krankheiten des Blutes H60-H95 Krankheiten des Ohres und des Warzenfortsatzes

P00-P96 Best. Zustände nit Ursprung i. d. Perinatalperiode

L00-L99 Krankheiten der Haut und der Unterhaut H00-H59 Krankheiten des Auges und der Augenanhangsgebilde D00-D48 Übrige Neubildungen

E00-E90 Endokrine, Ernährungs- und Stoffwechselkrankheiten

A00-B99 Bestimmte infektiöse und parasitäre Krankheiten

Z00-Z99 Faktoren, die zur Inanspruchn. d. Gesundheitsw. führen

G00-G99 Krankheiten des Nervensystems R00-R99 Symptome und abnorme klinische und Laborbefunde, ao. n. k. l. 000-099 Schwangerschaft, Geburt und Wochenbett

N00-N99 Krankheiten des Urogenitalsystems

F00-F99 Psychische und Verhaltensstörungen

J00-J99 Krankheiten des Atmungssystems

C00-C97 Bösartige Neubildungen

M00-M99 Krankh. d. Muskel-Skelett-Systems u. d. Bindegewebes

K00-K93 Krankheiten des Verdauungssystems

S00-T98 Verletzungen und Vergiftungen 100-199 Krankheiten des Kreislaufsystems

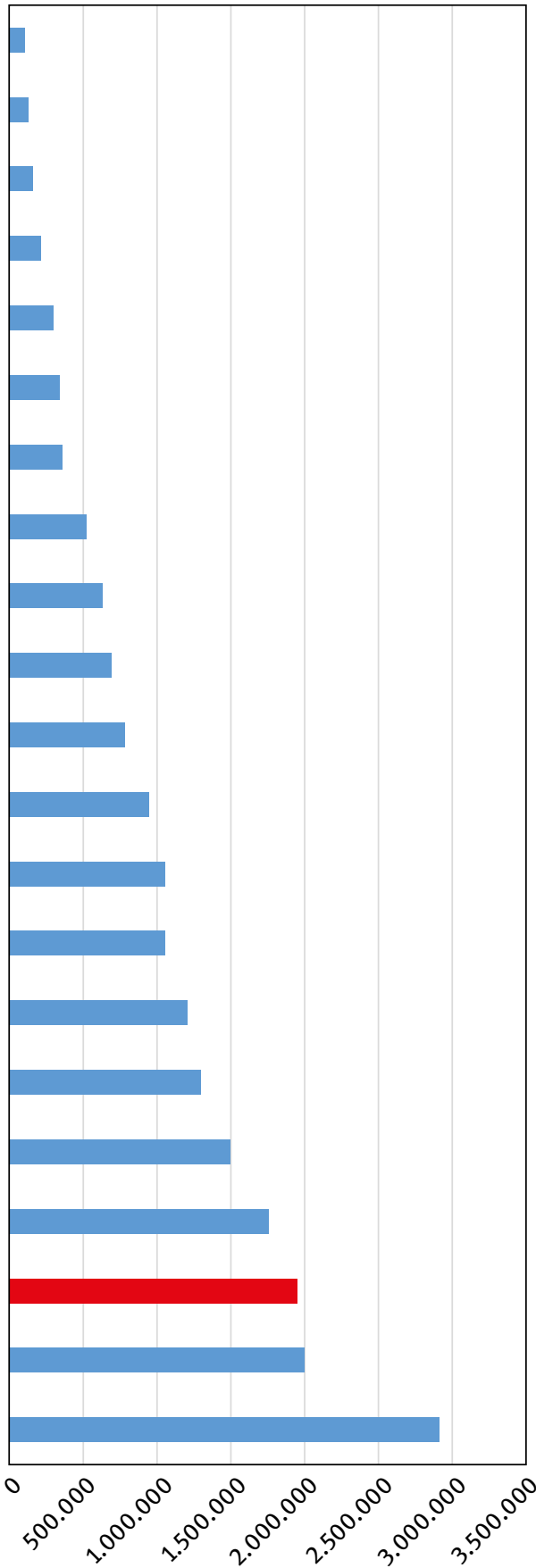

Abb. 1.1: Absolute Fallzahlen stationärer Behandlungsfälle 2017 (eigene Darstellung in Anlehnung an das Statistische Bundesamt [1]). 
000-099 Schwangerschaft, Geburt und Wochenbett H60-H95 Krankheiten des Ohres und des Warzenfortsatzes H00-H59 Krankheiten des Auges und der Augenanhangsgebilde Z00-Z99 Faktoren, die zur Inanspruchn. d. Gesundheitsw. führen Q00-Q99 Angeb. Fehlbild., Deformitäten, Chromosomenanomalien P00-P96 Best. Zustände mit Ursprung i.d. Perinatalperiode D50-D90 Krankheiten des Blutes D00-D48 Übrige Neubildungen

L00-L99 Krankheiten der Haut und der Unterhaut F00-F99 Psychische und Verhaltensstörungen M00-M99 Krankh. d. Muskel-Skelett-Systems und d. Bindegewebes G00-G99 Krankheiten des Nervensystems R00-R99 Symptome und abnorme klinische und Laborbefunde, ao. n. k. I.

E00-E90 Endokrine, Ernährungs- und Stoffwechselkrankheiten N00-N99 Krankheiten des Urogenitalsystems S00-T98 Verletzungen und Vergiftungen

A00-B99 Bestimmte infektiöse und parasitäre Krankheiten

K00-K93 Krankheiten des Verdauungssystems

J00-J99 Krankheiten des Atmungssystems C00-C97 Bösartige Neubildungen 100-199 Krankheiten des Kreislaufsystems

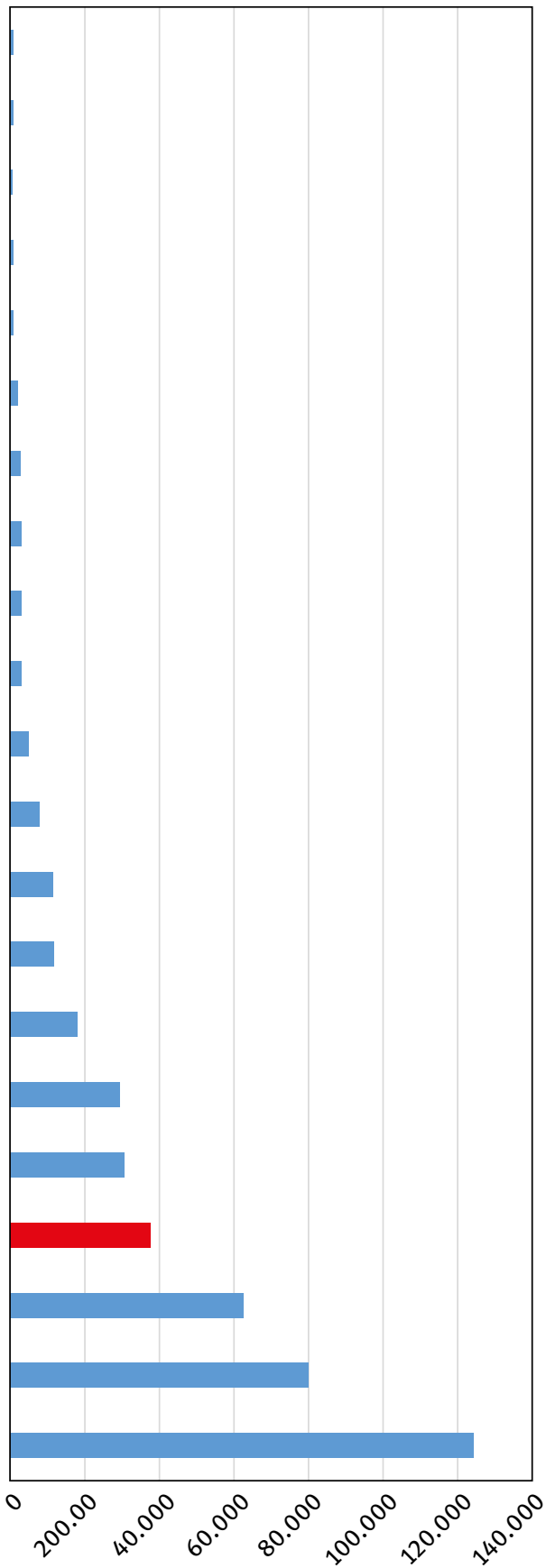

Abb. 1.2: Sterbefälle 2017 (eigene Darstellung in Anlehnung an das Statistische Bundesamt [2]). 


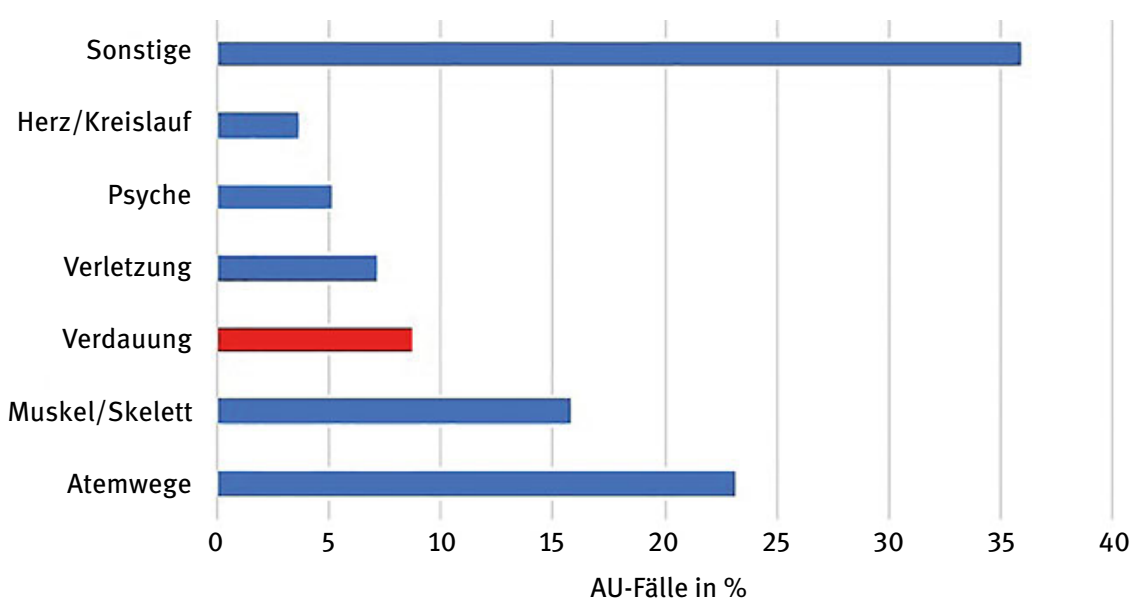

Abb. 1.3: Anteil der Krankheitsarten an Arbeitsunfähigkeitsfällen in Deutschland bis 2017 [3].

nahe, dass diese Kosten weiter gestiegen sind. Von 2008 bis 2015 stiegen auch die durchschnittlichen Krankheitskosten für Krankheiten des Verdauungssystems von 450 Euro auf 510 Euro pro Einwohner. Der Anteil an den Gesamtkosten liegt bei $12 \%$. Lediglich die Kosten für die Erkrankungen des Kreislaufsystems und der Psyche sind höher (Abb. 1.4) [4].

Dabei stellen die bisher genannten Zahlen nur einen Teilbereich dar, da auch die malignen Erkrankungen von Magen, Darm, Bauchspeicheldrüse, Galle und Leber (ICD C15-26, 329.174 Fälle 2017), die infektiösen Darmkrankheiten (ICD A00-A09, 251.726 Fälle 2017), zu denen zum Beispiel Infektionen durch Salmonellen, Campylobacter, Noroviren oder Clostridien gehören, sowie der Bereich der Virushepatitiden, insbesondere der Hepatitis B und C (ICD B15-B19, 3.860 Fälle 2017), in das Gebiet der Gastroenterologie fallen. Allein die Tumorerkrankungen des Magen-Darm-Traktes sind nicht nur die häufigsten bösartigen Erkrankungen in Deutschland, sie machen auch den größten Anteil der Sterbefälle aus (Abb. 1.5, Abb. 1.6).

Während jedoch für die benignen gastroenterologischen Erkrankungen in der letzten Dekade eine etwa 5\%ige Zunahme zu verzeichnen ist, ist die Zahl der malignen Erkrankungsfälle seit 2000 rückläufig und von über 410.000 auf aktuell 330.000 Patienten gesunken [1]. Verantwortlich für diesen Trend ist unter anderem die Einführung der gesetzlichen Früherkennung von Darmkrebs, die, ebenso wie die Innovationen in der Hepatitis-Therapie, ein herausragendes Beispiel für gastroenterologische Prävention darstellt [5].

In den folgenden Kapiteln werden die medizinischen Aspekte, die epidemiologischen Daten und die Kosten gastroenterologischer Erkrankungen dargestellt. Jedes einzelne Kapitel enthält eine Liste „offener Fragen“, die den aktuellen Bedarf an wissenschaftlichen Studien, medizinischen Innovationen und gesundheitsökonomi- 


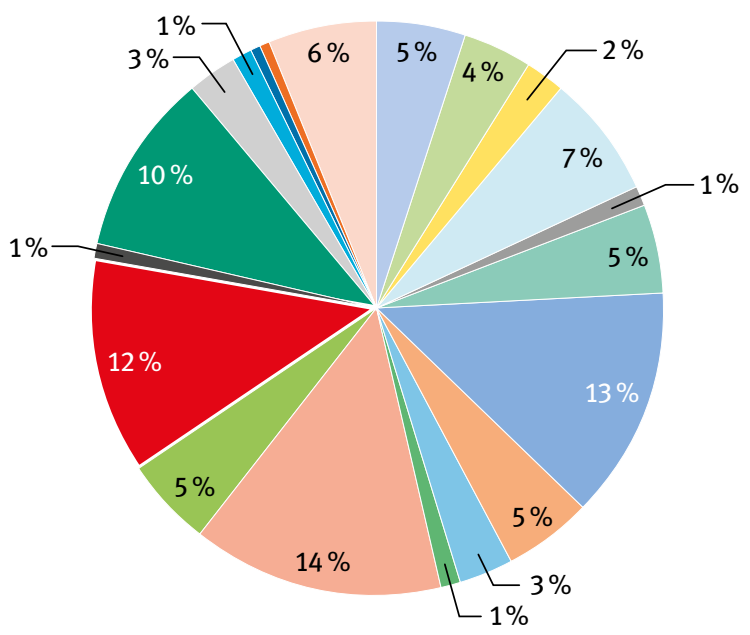

A00-B99 Bestimmte infektiöse und parasitäre Krankheiten

D50-D90 Kranheiten des Blutes

F00-F99 Psychische und Verhaltensstörungen

H00-H59 Krankheiten des Auges und der Augenanhangsgebilde

100-199 Krankheiten des Kreislaufsystems

K00-K93 Krankheiten des Verdauungssystems

M00-M99 Krankh. des Muskel-Skelett-Systems und des Bindegewebes

000-M99 Schwangerschaft, Geburt und Wochenbett

Q00-Q99 Angeborene Fehlbild., Deformitäten, Chromosomenanomalien

S00-T98 Verletzungen und Vergiftungen

C00-D48 Neubildungen

E00-E90 Endokrine, Ernährungs- und Stoffwechselkrankheiten

G00-G99 Krankheiten des Nervensystems

H60-H95 Krankheiten des Ohres und des Warzenfortsatzes

J00-J99 Krankheiten des Atmungssystems

L00-L99 Krankheiten der Haut und der Unterhaut

N00-N99 Krankheiten des Urogenitalsystems

- P00-P96 Bestimmte Zustände mit Ursprung i. d. Perinatalperiode

R00-R99 Symptome und abnorme klin. und Labor befunde, ao. n. kl.

Abb. 1.4: Krankheits-

kosten je Einwohner

Z00-Z99 Faktoren, die zur Inanspruchn. des Gesundheitswesens führen

in \% 2015 [4].

schen Verbesserungen darlegt. Insgesamt ist die verfügbare Datenlage für Deutschland als mangelhaft einzustufen, da in vielen Bereichen aussagekräftige Studien insbesondere zu der Epidemiologie und den Kosten fehlen.

Das Weißbuch beginnt mit den anatomisch gegliederten Themengebieten des unteren und oberen Gastrointestinaltrakts, der Leber, der Galle und des Pankreas. Organübergreifend folgen die Kapitel gastrointestinale Infektionen, maligne gastrointestinale Erkrankungen und Endoskopie. Ein eignes Kapitel ist dem Thema Prävention gewidmet und stellt den aktuellen Stand und die zukünftigen, herausragenden Möglichkeiten gastrointestinaler präventiver Maßnahmen dar. Ein weiteres Kapitel beschäftigt sich mit der Gastroenterologie in der Niederlassung, die in besonderem 
C15-C26 Bösartige Neubildungen der Verdauungsorgane

C30-C36 Bösartige Neubildungen der Atmungsorgane und sonstiger intrathorakaler Organe

C30-C96 Bösartige Neubild. des lymphatischen, blutbildenden und verwandten Gewebes, als ...

C64-C68 Bösartige Neubildungen der Harnorgane

C76-C80 Bösartige Neubild. ungenau bezeichneter, sekundärer u. nicht näher bezeichneter ...

C50-C50 Bösartige Neubildungen der Brustdrüse

C43-C44 Melanom und sonstige bösartige Neubildungen der Haut

C60-C63 Bösartige Neubildungen der männlichen Genitalorgane

C51-C58 Bösartige Neubildungen der weiblichen Genitalorgane

C00-C14 Bösartige Neubildungen der Lippe, der Mundhöhle und des Pharynx

C69-C72 Bösartige Neubild. des Auges, des Gehirns und sonstiger Teile des Zentralnervensyst.

C45-C49 Bösartige Neubild. des mesothelialen Gewebes und des Weichteilgewebes

C73-C75 Bösartige Neubild. der Schilddrüse und sonstiger endokriner Drüsen

C40-C41 Bösartige Neubildungen des Knochens und des Gelenkknorpels

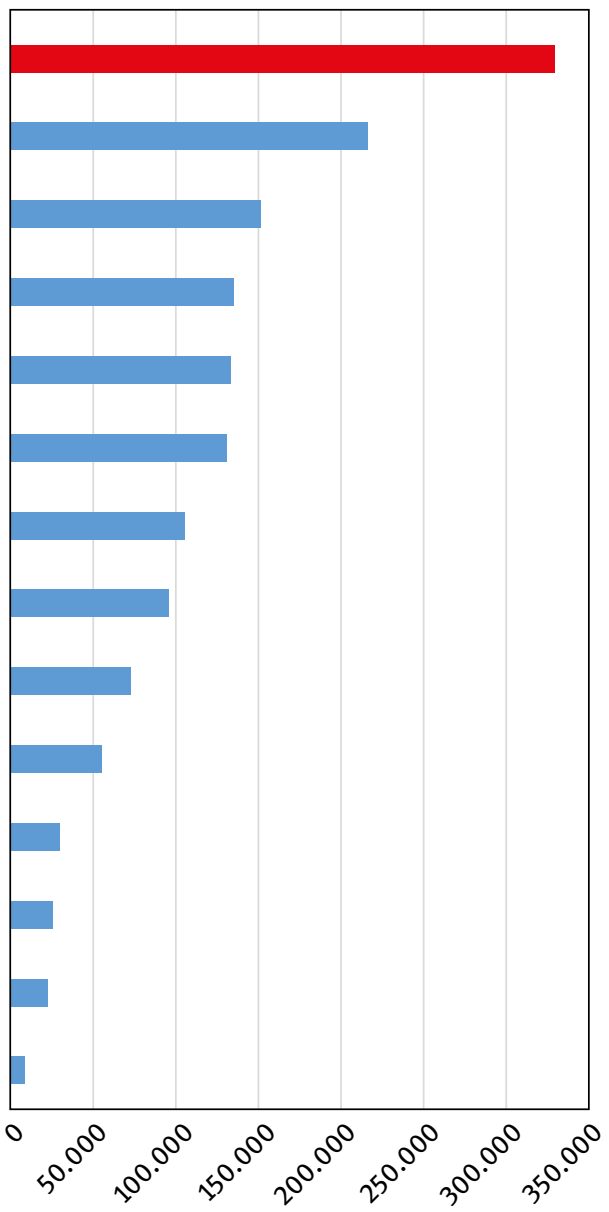

Abb. 1.5: Absolute Fallzahlen stationärer Behandlungsfälle bei malignen Erkrankungen 2017 (eigene Darstellung in Anlehnung an das Statistische Bundesamt) [1].

Maße an der Umsetzung präventiver Maßnahmen beteiligt ist. Auf dieser Wissensgrundlage wird abschließend auf die Perspektiven der gastroenterologischen Weiterbildung, Forschung und Lehre eingegangen. 
C15-C26 Bösartige Neubildungen der Verdauungsorgane

C30-C36 Bösartige Neubildungen der Atmungsorgane und sonstiger intrathorakaler Organe

C30-C96 Bösartige Neubild. des lymphatischen, blutbildenden und verwandten Gewebes, als ...

C76-C80 Bösartige Neubild. ungenau bezeichneter, sekundärer $u$. nicht näher bezeichneter ...

C50-C50 Bösartige Neubildungen der Brustdrüse

C64-C68 Bösartige Neubildungen der Harnorgane

C51-C58 Bösartige Neubildungen der weiblichen Genitalorgane

C60-C63 Bösartige Neubildungen der männlichen Genitalorgane

C00-C14 Bösartige Neubildungen der Lippe, der Mundhöhle und des Pharynx

C69-C72 Bösartige Neubild. des Auges, des Gehirns und sonstiger Teile des Zentralnervensyst.

C45-C49 Bösartige Neubild. des mesothelialen

Gewebes und des Weichteilgewebes

C43-C44 Melanom und sonstige bösartige Neubildungen der Haut

C73-C75 Bösartige Neubild. der Schilddrüse und sonstiger endokriner Drüsen

C40-C41 Bösartige Neubildungen des Knochens und des Gelenkknorpels

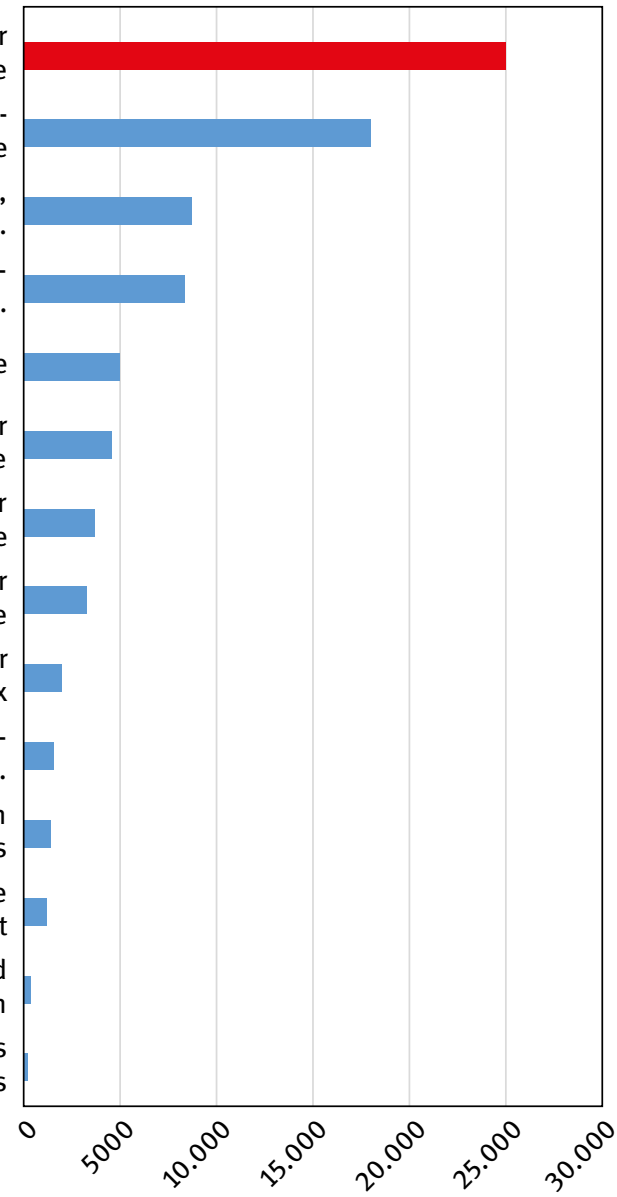

Abb. 1.6: Sterbefälle nach malignen Erkrankungen in deutschen Krankenhäusern 2017 (eigene Darstellung in Anlehnung an das Statistische Bundesamt) [1].

\section{Literatur}

[1] Statistisches Bundesamt. Krankenhausstatistik - Diagnosedaten der Patienten und Patientinnen in Krankenhäusern. www.gbe-bund.de. Zugriff: 20.02.2019.

[2] Statistisches Bundesamt. Todesursachenstatistik 2017. www.gbe-bund.de. Zugriff: 20.02.2019.

[3] AOK-Bundesverband und Wissenschaftliches Institut der AOK(WIdO). Fehlzeiten-Report 2018. https://aok-bv.de/presse/pressemitteilungen/2018/index_20972.html. Zugriff: 20.02.2019.

[4] Statistisches Bundesamt. Krankheitskostenrechnung 2015. www.gbe-bund.de. Zugriff: 20.02.2019.

[5] Brenner H, Kloor M, Pox CP. Colorectal cancer. The Lancet. 2014;383:1490-502. 
